\begin{tabular}{|c|l|}
\hline Title & Remarks on modified improved Boussinesq equations in one space dimension \\
\hline Author(s) & Cho, Yonggeun; Ozawa, Tohru \\
\hline Citation & Hokkaido University Preprint Series in Mathematics, 723, 1-15 \\
\hline Issue Date & 2005 \\
\hline DOI & 10.14943/83873 \\
\hline Doc URL & http://hdl.handle.net/2115/69531 \\
\hline Type & bulletin (article) \\
\hline File Information & pre723.pdf \\
\hline
\end{tabular}

Instructions for use 


\title{
REMARKS ON MODIFIED IMPROVED BOUSSINESQ EQUATIONS IN ONE SPACE DIMENSION
}

\author{
YONGGEUN CHO AND TOHRU OZAWA
}

\begin{abstract}
We study the existence and scattering of global small amplitude solutions to modified improved Boussinesq equations in one dimension with nonlinear term $f(u)$ behaving as a power $u^{p}$ as $u \rightarrow 0$. Solutions in $H^{s}$ space are considered for all $s>0$. According to the value of $s$, the power nonlinearity exponent $p$ is determined. Liu [15] obtained the minimum value of $p$ greater than 8 at $s=\frac{3}{2}$ for sufficiently small Cauchy data. In this paper, we prove that $p$ can be reduced to be greater than $\frac{9}{2}$ at $s>\frac{8}{5}$ and the corresponding solution $u$ has the time decay such as $\|u(t)\|_{L^{\infty}}=O\left(t^{-\frac{2}{5}}\right)$ as $t \rightarrow \infty$. We also prove nonexistence of nontrivial asymptotically free solutions for $1<p \leq 2$ under vanishing condition near zero frequency on asymptotic states.
\end{abstract}

\section{INTRODUCTION}

We consider the following initial value problem for the one dimensional generalized IMBq equation (Modified Improved Boussinesq equation):

$$
\begin{gathered}
u_{t t}-u_{x x t t}-u_{x x}=(f(u))_{x x}, \quad(x, t) \in \mathbb{R} \times(0,+\infty), \\
u(x, 0)=\varphi(x), \quad u_{t}(x, 0)=\psi(x), \quad x \in \mathbb{R},
\end{gathered}
$$

where $f \in C^{k}(\mathbb{C})$ in the real sense and $\left|f^{(l)}(u)\right| \lesssim|u|^{p-l}$ for $0 \leq l \leq k \leq p$ and $p>1$. By Duhamel's principle, the solution $u$ can be written as

$$
u(x, t)=\left(\partial_{t} S(t) \varphi\right)(x)+(S(t) \psi)(x)+\int_{0}^{t} T\left(t-t^{\prime}\right) f\left(u\left(t^{\prime}\right)\right) d t^{\prime} .
$$

Here $T(t)=S(t)\left(I-\partial_{x}^{2}\right)^{-1} \partial_{x}^{2}$ and

$$
\begin{gathered}
\left(\partial_{t} S(t) \varphi\right)(x)=\frac{1}{2 \pi} \int_{\mathbb{R}} e^{i x \xi} \cos \left(\frac{t \xi}{\sqrt{1+\xi^{2}}}\right) \widehat{\varphi}(\xi) d \xi \\
(S(t) \psi)(x)=\frac{1}{2 \pi} \int_{\mathbb{R}} e^{i x \xi} \sin \left(\frac{t \xi}{\sqrt{1+\xi^{2}}}\right) \frac{\sqrt{1+\xi^{2}}}{\xi} \widehat{\psi}(\xi) d \xi
\end{gathered}
$$

where $\widehat{\varphi}(\xi)=\mathcal{F}(\varphi)(\xi)=\int_{\mathbb{R}} e^{-i x \cdot \xi} \varphi(x) d x$ is the Fourier transform of $\varphi$.

The generalized IMBq equation governs the various physical models like nonlinear wave in weakly dispersive medium (in this case $f(u)=u^{2}[3,13,16]$ ) and longitudinal variation wave in elastic $\operatorname{rod}\left(f(u)=u^{3}\right.$ or $\left.u^{5}[10]\right)$, etc. For the local

2000 Mathematics Subject Classification. 35Q53, 47J35.

Key words and phrases. IMBq equation, small amplitude solution, global existence, scattering. The first author is JSPS Fellow. 
or global existence of solution of IMBq equations, see $[4,5,6,8,15]$, and for the small amplitude solution and scattering, see [7, 15].

In this paper, the small amplitude solution and scattering to the nonlinear problem (1.1) are considered in one dimensional case. Our main concerns are to provide the lower bound of nonlinearity $p$ for the global existence of solution and scattering according to the regularity of initial data, and also the upper bound of $p$ for the nonexistence of nontrivial asymptotically free solutions. The methods below can be applied to the high dimensional case as well without any difficulty. For this see the section 3.3 Remarks below.

To state our main results, let us define a function space $X_{\delta}^{s, \theta}$ by

$$
X_{\rho}^{s, \theta}=\left\{v:\|v\|\left\|_{s, \theta} \equiv \sup _{t>0}(1+t)^{\theta}\right\| v\left\|_{L^{\infty}}+\sup _{t>0}\right\| D^{s} v \|_{L^{2}} \leq \rho\right\}
$$

and $D^{\alpha} L^{1}$ and $D L^{2}$ by $\left\{\varphi: D^{-\alpha} \varphi \in L^{1}\right\}$ and $\left\{\varphi: D^{-1} \varphi \in L^{2}\right\}$ respectively, where $D=\sqrt{-\partial_{x}^{2}}$. We use the usual Sobolev spaces $W_{r}^{s}$ and $H^{s}$ with the norms

$$
\|\varphi\|_{W_{r}^{s}}=\|\varphi\|_{L^{r}}+\left\|D^{s} \varphi\right\|_{L^{r}}, \quad\|\varphi\|_{H^{s}}=\|\varphi\|_{W_{2}^{s}} .
$$

The first result is on the following global existence for small data.

Theorem 1.1. Let $s, p, \alpha$ be numbers such that $k \geq s>2-\frac{4}{r}$

$$
p>\max \left(\frac{4 r-4}{r-2}, \frac{r+8}{4}\right) \quad \text { and } \quad \alpha=\max \left(\frac{3(r-2)}{2 r}, \frac{r-2}{4}\right)
$$

for $2<r<\infty$. Suppose that the data $(\varphi, \psi)$ satisfy the regularity condition

$$
(\varphi, \psi) \in\left(L^{1} \cap D^{\max (0, \alpha-1)} L^{1} \cap W_{r^{\prime}}^{s} \cap H^{s}\right) \times\left(L^{1} \cap D^{\alpha} L^{1} \cap D L^{2} \cap W_{r^{\prime}}^{s} \cap H^{s}\right)
$$

and the smallness condition

$$
\begin{aligned}
\|\varphi\|_{L^{1}} & +\left\|D^{-\max (0, \alpha-1)} \varphi\right\|_{L^{1}}+\|\varphi\|_{W_{r^{\prime}}^{s}}+\|\varphi\|_{H^{s}} \\
& +\|\psi\|_{L^{1}}+\left\|D^{-\alpha} \psi\right\|_{L^{1}}+\left\|D^{-1} \psi\right\|_{L^{2}}+\|\psi\|_{W^{s} r^{\prime}}+\|\psi\|_{H^{s}} \leq \delta .
\end{aligned}
$$

Then if $\delta$ is sufficiently small, then there exists a unique global solution $u \in$ $C^{1}\left([0, \infty) ; L^{\infty} \cap H^{s}\right)$ of $(1.2)$ and small positive number $\rho$ depending only on $r, \delta$ such that

$$
\left\|\left|u\left\|_{s, \min \left(\frac{r-2}{2 r}, \frac{4}{r}\right)}+\right\|\right| \mid u_{t}\right\|_{s, \min \left(\frac{r-2}{2 r}, \frac{4}{r}\right)} \leq \rho .
$$

The next is on the scattering.

Theorem 1.2. Let $u$ be the solution of (1.2) as in Theorem 1.1. Then there exist functions $\varphi^{+}$and $\psi^{+}$in $H^{s}$ such that

$$
\left\|u(t)-u^{+}(t)\right\|_{H^{s}}+\left\|u_{t}(t)-u_{t}^{+}(t)\right\|_{H^{s}}=O\left(t^{-(p-1) \min \left(\frac{r-2}{2 r}, \frac{4}{r}\right)+1}\right),
$$

where $u^{+}$is the unique solution of linear homogeneous equation

$$
\begin{gathered}
u_{t t}^{+}-u_{t t x x}^{+}-u_{x x}^{+}=0, \\
u^{+}(0)=\varphi^{+}, u_{t}^{+}(0)=\psi^{+} .
\end{gathered}
$$


The minimum values of $p$ can be chosen to be greater than $\frac{9}{2}$ at $r=10$ and then $\alpha=2$ and $s>\frac{8}{5}$. We also have that $\sup _{t>0}(1+t)^{\frac{2}{5}}\|u(t)\|_{L^{\infty}}<\infty$. If we choose $r=6$, then we can take the values $p, \alpha, s$ as $p>5, \alpha=1$ and $s>\frac{4}{3}$, and also have that $\sup _{t>0}(1+t)^{\frac{1}{3}}\|u(t)\|_{L^{\infty}}<\infty$. Thus Theorems 1.1 and 1.2 contain the physical situation $p=5$ and also give slight improvements of the previous result [15] in which the global existence was established for $p>8$ at $s=\frac{3}{2}$ and the value of $s$ should be greater than $\frac{1}{2}$ for the scattering. Moreover, even if $s \leq \frac{1}{2}$ (this can occur for $2<r<\frac{8}{3}$ ), the global existence can be established and time decay estimate can be obtained without resort to the Sobolev embedding $H^{s} \hookrightarrow L^{\infty}$ for $s>\frac{1}{2}$.

For the purpose of improvement, we use the stationary phase method and Young's inequality $\left(\|u * v\|_{L^{\infty}} \leq\|u\|_{L^{r}}\|v\|_{L^{r^{\prime}}}\right)$ for the dyadically localized kernel estimate of high frequency part of $\partial_{t} S, S$ and $T$ instead of integration estimate used in [15] and [7]. For the kernel estimate, the condition $\varphi, \psi \in W_{r^{\prime}}^{s}$ is used. We also use van der Corput type estimate for medium frequency part of the operators similar to the one in $[7,15]$. To obtain an estimate for low frequency part, the condition $\psi \in D^{\alpha} L^{1} \cap D L^{2}$ is necessary. For the details, see Section 2.2 below.

In [7], the same problem was considered and some extended results were obtained but the results should be corrected because the authors overlooked the bad behavior of low frequency part of $S(t) \psi$ at near zero frequency which causes troubles in $L^{\infty}$ and $H^{s}$ estimates.

In view of Theorem 1.1, if $r=10$ and hence $\varphi \in D\left(L^{1} \cap L^{2}\right)$ and $\psi \in D^{2}\left(L^{1} \cap L^{2}\right)$, then for $p>\frac{9}{2}$, it can be easily shown that $\left(u, u_{t}\right) \in L^{\infty}\left(0, \infty ; D L^{2} \times D^{2} L^{2}\right)$ by the decay estimate $\sup _{t>0}(1+t)^{\frac{2}{5}}\|u(t)\|_{L^{\infty}}<\infty$ and the scattering $\left\|u(t)-u^{+}(t)\right\|_{L^{2}}=$ $O\left(t^{-\frac{2}{5}}\right)$. On the other hand, the following theorem shows that there is no nontrivial asymptotically free solution $u$ with $\left\|u(t)-u^{+}(t)\right\|_{L^{2}}=O\left(t^{-\varepsilon}\right)$, if $p$ is small and $\left(u, u_{t}\right) \in L^{\infty}\left(0, \infty ; D L^{2} \times D^{2} L^{2}\right)$.

Theorem 1.3. Let $1<p \leq 2$ and suppose that $\operatorname{Re}(f(u) \bar{u}) \geq c|u|^{p+1}$ for some positive constant c. Let $u$ be a smooth solution $u$ to $(1.1)$ with $\left(u, u_{t}\right) \in L^{\infty}\left(0, \infty ; D L^{2} \times\right.$ $\left.D^{2} L^{2}\right)$ and $\left(\varphi^{+}, \psi^{+}\right)$be a pair of smooth functions with compactly supported $\widehat{\varphi^{+}}$and $\widehat{\psi^{+}}$in $\mathbb{R} \backslash\{0\}$. Suppose that

$$
\left\|u(t)-u^{+}(t)\right\|_{L^{2}}=O\left(t^{-\varepsilon}\right) \quad \text { as } \quad t \rightarrow \infty
$$

for some $\varepsilon>0$, where $u^{+}$is the free solution to the linear problem (1.4). Then $u=u^{+}=0$.

The theorem above shows that IBq equation (corresponding to the physical situation $f(u)=u^{2}$ ) does not have nontrivial asymptotically free solution. But it remains open whether the theorem is true for $p>2$ in one dimensional case or not. For the proof, we use an analogous argument to the one of Glassey [11] that 
$H(t)=\operatorname{Re} \int\left(D^{-1} u_{t} \overline{D^{-1} u^{+}}-D^{-1} u_{t}^{+} \overline{D^{-1} u}\right) d x$ is uniformly bounded but under the conditions stated in Theorem $1.3 \frac{d}{d t} H(t) \geq \frac{c}{t}$ and hence a contradiction occurs. For related topics, see $[1,17,19]$.

If not specified, throughout this paper, the notation $A \lesssim B$ and $A \gtrsim B$ denote $A \leq C B$ and $A \geq C^{-1} B$, respectively. Positive constants $C$ vary line by line and depend only on $r$ and $f$. $A \sim B$ means that both $A \lesssim B$ and $A \gtrsim B$ hold.

\section{Preliminaries}

2.1. Linear estimates. First, we introduce an estimate of oscillatory integral.

Lemma 2.1. For $R, t>1$ and $0<\varepsilon<1$, we have

$$
\begin{gathered}
\sup _{x \in \mathbb{R}}\left|\int_{\varepsilon<|\xi|<R} e^{i\left(x \xi \pm \frac{t \xi}{\sqrt{1+\xi^{2}}}\right)} F(\xi) \frac{d \xi}{|\xi|^{m}}\right| \\
\lesssim \varepsilon^{-m} \max \left(\varepsilon^{-\frac{1}{2}}, R^{2}\right) t^{-\frac{1}{2}}\left(\|F\|_{L^{\infty}(\varepsilon, R)}+\int_{\varepsilon}^{R}\left|F^{\prime}(\xi)\right| d \xi\right),
\end{gathered}
$$

where $m \geq 0$ and $F \in C^{1}[\varepsilon, R]$.

Proof. A direct application of van der Corput lemma [18] yields readily the proof. For the case $m=0$, see Lemma 4.3 in [15] or Lemma 2.2 in [7].

Let us choose a Littlewood-Paley function $\eta$ with and define a frequency projection operator $P_{N}$ for a dyadic number $N$ by

$$
P_{N} \phi(x)=\frac{1}{2 \pi} \int e^{i x \xi} \eta\left(\frac{\xi}{N}\right) \widehat{\phi}(\xi) d \xi .
$$

And we also denote $P_{\leq \varepsilon} \phi, P_{\geq N_{0}} \phi$ and $P_{\varepsilon<\cdot<N_{0}} \phi$ by

$$
\begin{aligned}
P_{\leq \varepsilon} \phi & =\sum_{N \leq \varepsilon} P_{N} \phi \quad \text { (low frequency part), } \\
P_{\geq N_{0}} \phi & =\sum_{N \geq N_{0}} P_{N} \phi \quad \text { (high frequency part), } \\
P_{\varepsilon<<N_{0}} \phi & =\sum_{\varepsilon<N<N_{0}} P_{N} \phi \quad \text { (medium frequency part). }
\end{aligned}
$$

We choose $\eta$ so that $P_{N}=P_{N-2<\cdot<N+2} P_{N}$.

Lemma 2.2. Let $2<r<\infty$ and $s>2-\frac{4}{r}$. Then for any $\varphi \in L^{1} \cap D^{\min (0, \alpha-1)} L^{1} \cap$ $W_{r^{\prime}}^{s}$ with $\alpha=\frac{r-2}{4}$ we have

$$
\left\|\partial_{t} S(t) \varphi\right\|_{L^{\infty}} \lesssim(1+t)^{-\left(\frac{1}{2}-\frac{1}{r}\right)}\left(\|\varphi\|_{L^{1}}+\left\|D^{-\min (0, \alpha-1)} \varphi\right\|_{L^{1}}+\left\|D^{s} \varphi\right\|_{L^{r^{\prime}}}\right) .
$$

Proof. Taking $P_{N}$ to $\partial_{t} S(t)$ and using change of variable, we have

$$
\begin{aligned}
\left|P_{N}\left(\partial_{t} S(t) \varphi\right)(x)\right| & =\left|\int e^{i N x \xi} \cos \left(N^{-2} t \omega_{N}(\xi)\right) \eta(\xi) \mathcal{F}\left[P_{N-2<\cdot<N+2} \varphi\left(\frac{\dot{N}}{N}\right)\right](\xi) d \xi\right| \\
& \lesssim\left|K\left(N(\cdot), N^{-2} t\right)\right| *\left|P_{N-2<\cdot<N+2} \varphi\left(\frac{\dot{\bar{N}}}{N}\right)\right|,
\end{aligned}
$$


where $\omega_{N}(\xi)=\frac{N^{2} \xi}{\sqrt{N^{-2}+\xi^{2}}}$ and

$$
\begin{aligned}
K\left(N x, N^{-2} t\right) & =\frac{1}{2 \pi} \int e^{i N x \xi} \cos \left(N^{-2} t \omega_{N}\right) \eta(\xi) d \xi \\
& =\frac{1}{4 \pi} \int\left(e^{i\left(N x \xi+N^{-2} t \omega_{N}\right)}+e^{i\left(N x \xi-N^{-2} t \omega_{N}\right)}\right) \eta(\xi) d \xi
\end{aligned}
$$

Since $\left|\omega_{N}^{\prime}(\xi)\right| \sim\left|\omega_{N}^{\prime \prime}(\xi)\right| \sim 1$ for sufficiently large $N$ and $|\xi| \sim 1$, by the method of stationary and non-stationary phase [18], we have

$$
\begin{aligned}
& \left|P_{N}\left(\partial_{t} S(t) \varphi\right)(x)\right| \lesssim \int\left(1+N^{-2} t+N|x-y|\right)^{-\frac{1}{2}}\left|P_{N-2<\cdot<N+2} \varphi\left(\frac{y}{N}\right)\right| d y \\
& \lesssim\left\|\left(1+N^{-2} t+N|\cdot|\right)^{-\frac{1}{2}}\right\|_{L^{r}}\left\|P_{N-2<\cdot<N+2} \varphi\left(\frac{\cdot}{N}\right)\right\|_{L^{r^{\prime}}} \\
& \lesssim\left(1+N^{-2} t\right)^{-\frac{1}{2}}\left(\frac{N}{1+N^{-2} t}\right)^{-\frac{1}{r}} N^{\frac{1}{r^{\prime}}}\left\|P_{N-2<\cdot<N+2} \varphi\right\|_{L^{r^{\prime}}} \\
& \lesssim(1+t)^{-\left(\frac{1}{2}-\frac{1}{r}\right)} N^{2-\frac{4}{r}}\left\|P_{N-2<\cdot<N+2} \varphi\right\|_{L^{r^{\prime}}}
\end{aligned}
$$

Thus using $s>2-\frac{4}{r}$, we deduce that for large $N_{0}$ and any $2<r<\infty$

$$
\begin{aligned}
\left\|P_{\geq N_{0}} \partial_{t} S(t) \varphi\right\|_{L^{\infty}} & \lesssim \sum_{N \geq N_{0}}(1+t)^{-\left(\frac{1}{2}-\frac{1}{r}\right)} N^{2-\frac{4}{r}}\left\|P_{N-2<\cdot<N+2} \varphi\right\|_{L^{r^{\prime}}} \\
& \lesssim(1+t)^{-\left(\frac{1}{2}-\frac{1}{r}\right)}\left\|P_{\geq 1} \varphi\right\|_{B_{r^{\prime}, 2}^{s}} \\
& \lesssim(1+t)^{-\left(\frac{1}{2}-\frac{1}{r}\right)}\left\|D^{s} \varphi\right\|_{L^{r^{\prime}}}
\end{aligned}
$$

Here $B_{r^{\prime}, 2}^{s}$ is the Besov space with norm for $s>0,1<r^{\prime}<\infty$ by

$$
\|\varphi\|_{B_{r^{\prime}, 2}^{s}}=\|\varphi\|_{L^{r^{\prime}}}+\left(\sum_{N: \text { dyadic number }} N^{2 s}\left\|P_{N} \varphi\right\|_{L^{r^{\prime}}}^{2}\right)^{\frac{1}{2}} .
$$

For the last inequality, we used the well-known embedding $W_{q}^{s} \hookrightarrow B_{q, 2}^{s}$ for $1<q \leq 2$ and the fact $\left\|P_{\geq 1} \varphi\right\|_{W_{r^{\prime}}^{s}} \lesssim\left\|D^{s} \varphi\right\|_{L^{r^{\prime}}}$ (see for instance [2]).

As for the medium frequency of $\partial_{t} S(t) \varphi$, using Lemma 2.1, we can easily show that for $t>1$

$$
\left\|P_{\varepsilon<\cdot<N_{0}} \partial_{t} S(t) \varphi\right\|_{L^{\infty}} \lesssim \max \left(\varepsilon^{-\frac{1}{2}}, N_{0}^{2}\right) t^{-\frac{1}{2}}\|\varphi\|_{L^{1}} .
$$

By Hausdorff-Young's inequality, we have

$$
\begin{aligned}
&\left\|P_{\leq \varepsilon} \partial_{t} S(t) \varphi\right\|_{L^{\infty}} \lesssim \varepsilon\|\varphi\|_{L^{1}}, \quad \text { if } \quad r \leq 6, \\
&\left\|P_{\leq \varepsilon} \partial_{t} S(t) \varphi\right\|_{L^{\infty}} \lesssim \varepsilon^{\alpha}\left\|D^{-(\alpha-1)} \varphi\right\|_{L^{1}}, \quad \text { if } \quad r>6 .
\end{aligned}
$$

Now let us choose $\varepsilon$ by $t^{-\frac{2}{r}}\left(\leq N_{0}^{-4}\right)$. Then since $\frac{2}{r} \geq \frac{1}{2}-\frac{1}{r}$ for $r \leq 6$ and $\frac{2 \alpha}{r} \geq \frac{1}{2}-\frac{1}{r}$ for $r>6$, from (2.2) and (2.3) we have for $t>N_{0}^{2 r}$

$$
\begin{aligned}
& \left\|P_{\leq N_{0}} \partial_{t} S(t) \varphi\right\|_{L^{\infty}} \lesssim t^{-\left(\frac{1}{2}-\frac{1}{r}\right)}\|\varphi\|_{L^{1}} \quad \text { for } \quad r \leq 6 \\
& \left\|P_{\leq N_{0}} \partial_{t} S(t) \varphi\right\|_{L^{\infty}} \lesssim t^{-\left(\frac{1}{2}-\frac{1}{r}\right)}\left(\|\varphi\|_{L^{1}}+\left\|D^{-(\alpha-1)} \varphi\right\|_{L^{1}}\right) \text { for } \quad r>6 .
\end{aligned}
$$


If $t \leq N_{0}^{2 r}$, then by another use of the method of non-stationary phase, we have

$$
\begin{aligned}
\left\|\partial_{t} S(t) \varphi\right\|_{L^{\infty}} & \leq\left\|P_{\leq N_{0}^{r}} \partial_{t} S(t) \varphi\right\|_{L^{\infty}}+\left\|P_{>N_{0}^{r}} \partial_{t} S(t) \varphi\right\|_{L^{\infty}} \\
& \lesssim\|\varphi\|_{L^{1}}+\sum_{N>N_{0}^{r}} N^{1-\frac{2}{r}}\left\|P_{N} \varphi\right\|_{L^{r^{\prime}}} \lesssim\|\varphi\|_{L^{1}}+\left\|D^{s} \varphi\right\|_{L^{r^{\prime}}}
\end{aligned}
$$

Combining this estimate, (2.1) and (2.4), we obtain for $\alpha=\frac{r-2}{4}$

$$
\left\|\partial_{t} S(t) \varphi\right\|_{L^{\infty}} \lesssim(1+t)^{-\left(\frac{1}{2}-\frac{1}{r}\right)}\left(\|\varphi\|_{L^{1}}+\left\|D^{-\min (0, \alpha-1)} \varphi\right\|_{L^{1}}+\left\|D^{s} \varphi\right\|_{L^{r^{\prime}}}\right)
$$

Lemma 2.3. Let $2<r<\infty$ and $s>2-\frac{4}{r}$. Then for any $\psi \in L^{1} \cap D^{\alpha} L^{1} \cap W_{r^{\prime}}^{s}$ with $\alpha=\frac{3(r-2)}{2 r}$, if $r \leq 6$ and $\alpha=\frac{r-2}{4}$, if $r>6$ we have

$$
\|S(t) \psi\|_{L^{\infty}} \lesssim(1+t)^{-\left(\frac{1}{2}-\frac{1}{r}\right)}\left(\|\psi\|_{L^{1}}+\left\|D^{-\alpha} \psi\right\|_{L^{1}}+\left\|D^{s} \varphi\right\|_{L^{r^{\prime}}}\right) .
$$

Proof. The proof for the high frequency part of $S(t)$ is almost the same as the one for $\partial_{t} S(t)$. Thus we consider only the low and medium frequency parts. With $\alpha$ as above, we have

$$
\left\|P_{\leq \varepsilon} S(t) \psi\right\|_{L^{\infty}} \lesssim \varepsilon^{\alpha}\left\|D^{-\alpha} \psi\right\|_{L^{1}} .
$$

On the other hand, for the medium frequency we have from Lemma 2.1 that

$$
\left\|P_{\varepsilon<\cdot<N_{0}} S(t) \psi\right\|_{L^{\infty}} \lesssim \max \left(1, \varepsilon^{-(1-\alpha)}\right) \max \left(\varepsilon^{-\frac{1}{2}}, N_{0}^{2}\right) t^{-\frac{1}{2}}\left\|D^{-\alpha} \psi\right\|_{L^{1}},
$$

if $t>1$. Now if we choose $\varepsilon=t^{-\frac{2}{(3-2 \alpha) r}}\left(\leq N_{0}^{-4}\right)$ for $r \leq 6$ and $t^{-\frac{2}{r}}\left(\leq N_{0}^{-4}\right)$ for $r>6$, then since $\frac{2 \alpha}{(3-2 \alpha) r} \geq \frac{1}{2}-\frac{1}{r}$ for $r \leq 6$ and $\frac{2 \alpha}{r} \geq \frac{1}{2}-\frac{1}{r}$, we have

$$
\|S(t) \psi\|_{L^{\infty}} \lesssim t^{-\left(\frac{1}{2}-\frac{1}{r}\right)}\left(\|\psi\|_{L^{1}}+\left\|D^{-\alpha} \psi\right\|_{L^{1}}+\left\|D^{s} \psi\right\|_{L^{r^{\prime}}}\right)
$$

for large $t$. If $t$ is small, then similarly to the estimate for $\partial_{t} S(t)$ we have

$$
\begin{aligned}
\|S(t) \psi\|_{L^{\infty}} & \leq\left\|P_{\leq N_{0}^{\beta}} S(t) \psi\right\|_{L^{\infty}}+\left\|P_{>N_{0}^{\beta}} S(t) \psi\right\|_{L^{\infty}} \\
& \lesssim\left\|D^{-\alpha} \psi\right\|_{L^{1}}+\left\|D^{s} \psi\right\|_{L^{r^{\prime}}},
\end{aligned}
$$

where $\beta=(3-2 \alpha) r$ for $r \leq 6$ and $\beta=r$ for $r>6$. We have just finished the proof of the lemma.

As a corollary of Lemmas 2.2 and 2.3, we have the following lemma.

Lemma 2.4. Let $2<r<\infty$ and $s>2-\frac{4}{r}$. Then for any $g(\cdot, t) \in L^{1} \cap W_{r^{\prime}}^{s}$, we have

$\left\|\int_{0}^{t} T\left(t-t^{\prime}\right) g\left(t^{\prime}\right) d t^{\prime}\right\|_{L^{\infty}} \lesssim \int_{0}^{t}\left(1+t-t^{\prime}\right)^{-\min \left(\frac{1}{2}-\frac{1}{r}, \frac{4}{r}\right)}\left(\left\|g\left(t^{\prime}\right)\right\|_{L^{1}}+\left\|D^{s} g\left(t^{\prime}\right)\right\|_{L^{r^{\prime}}}\right) d t^{\prime}$.

Proof. The only difference between $T(t)$ and $\partial_{t} S(t)$ consists in the lower frequency part. For this, we have

$$
\left\|P_{\leq \varepsilon} T(t) g\right\|_{L^{\infty}} \lesssim \varepsilon^{2}\|g\|_{L^{1}}
$$


Thus from the low and medium frequency estimate in the proof of Lemma 2.2, we deduce

$$
\left\|P_{\leq N_{0}} T(t) g\right\|_{L^{\infty}} \lesssim t^{-\min \left(\frac{1}{2}-\frac{1}{r}, \frac{4}{r}\right)}\|g\|_{L^{1}}
$$

for large $t$. This completes the proof.

2.2. Remarks on the linear estimates. In view of the proof of Lemmas 2.2 and 2.3 , it follows that if $D^{-(\alpha-1)} \varphi, D^{-\alpha} \psi \in L^{1}$ for $\alpha>1$, the range of $r$ can be extended up to $4 \alpha+2$ and hence the time decay of supremum norm becomes faster. This fact implies that if $\widehat{\varphi}$ and $\widehat{\psi}$ are zero near the origin and compactly supported, then the time decay rate can be taken by the maximal decay rate $\frac{1}{2}$. Thus we can expect that the scattering holds up to $p>3$ as the case of Schrödinger equation. But in Lemma 2.4, we were not able to obtain such decay because of the infinite speed of propagation which makes it impossible to use the zero frequency. We need more subtle estimate near zero frequency.

In Lemmas 2.2 and 2.3, we used the condition $D^{-\alpha} \psi \in L^{1}$ and $D^{-1} \psi \in L^{2}$ for some time decay of the supreme norm and uniform bound on time of Sobolev norm of $S \psi$, respectively. In [15], the condition $\left(1-\partial_{x}^{2}\right)^{\frac{1}{2}} D^{-1} \psi \in L^{1} \cap L^{2}$ was used. Actually, the condition $\left(1-\partial_{x}^{2}\right)^{\frac{1}{2}} D^{-1} \psi \in L^{2}$ is necessary for the energy conservation and momentum conservation. This type condition implies at least that $\widehat{\psi}$ should be zero at $\xi=0$. This vanishing condition at zero frequency turns out to be inevitable for the uniform bound because of the following fact: if $\widehat{\psi}=1$ if $|\xi|<1$ and 2 if $|\xi| \geq 2$, then for large $t$

$$
\|S(t) \psi\|_{L^{2}}^{2} \sim t \int \frac{1+\xi^{2} / t^{2}}{\xi^{2}}\left|\sin \left(\frac{\xi}{\sqrt{1+\xi^{2} / t^{2}}}\right)\right|^{2}|\widehat{\psi}(\xi / t)|^{2} d \xi \gtrsim t \int|\widehat{\psi}|^{2} d \xi \rightarrow \infty .
$$

Moreover, the vanishing condition is inevitable for the time decay. To see this, let $\psi$ be a smooth function such that $\widehat{\psi}=1$ if $|\xi|<1$ and $\widehat{\psi}=0$ if $|\xi|>2$. Then the limit $\lim _{t \rightarrow \infty} S(t) \psi(x)$ exists for all $x$ and the following holds

$$
\liminf _{t \rightarrow \infty}\|S(t) \psi\|_{L^{\infty}} \geq \frac{1}{2}
$$

For the proof let us choose a positive number $\theta$ smaller than $\frac{1}{3}$. Then by Lemma 2.1 with $m=1$ and $F(\xi)=\sqrt{1+\xi^{2}}\left(1-\widehat{\psi}\left(t^{\theta} \xi\right)\right) \widehat{\psi}(\xi)$, we have

$$
\left\|\int_{\mathbb{R}} e^{i x \xi} \sin \left(\frac{t \xi}{\sqrt{1+\xi^{2}}}\right) \frac{\sqrt{1+\xi^{2}}}{\xi}\left(1-\widehat{\psi}\left(t^{\theta} \xi\right)\right) \widehat{\psi}(\xi) d \xi\right\|_{L^{\infty}} \lesssim t^{\frac{3}{2} \theta-\frac{1}{2}} \rightarrow 0
$$

as $t \rightarrow \infty$. Thus for the proof of the estimate (2.5), it suffices to show that

$$
\frac{1}{2 \pi} \int_{\mathbb{R}} e^{i x \xi} \sin \left(\frac{t \xi}{\sqrt{1+\xi^{2}}}\right) \frac{\sqrt{1+\xi^{2}}}{\xi} \widehat{\psi}\left(t^{\theta} \xi\right) d \xi \rightarrow \frac{1}{2 i}
$$


uniformly on compact subsets of $\mathbb{R}$. Letting $\varepsilon=t^{-1}$, by change of variable, we have

$$
\begin{aligned}
& \frac{1}{2 \pi} \int_{\mathbb{R}} e^{i x \xi} \sin \left(\frac{t \xi}{\sqrt{1+\xi^{2}}}\right) \frac{\sqrt{1+\xi^{2}}}{\xi} \widehat{\psi}\left(t^{\theta} \xi\right) d \xi \\
= & \frac{1}{2 \pi} \int_{\mathbb{R}} e^{i \varepsilon x \xi} \sin \left(\frac{\xi}{\sqrt{1+\varepsilon^{2} \xi^{2}}}\right) \frac{\sqrt{1+\varepsilon^{2} \xi^{2}}}{\xi} \widehat{\psi}\left(\varepsilon^{1-\theta} \xi\right) d \xi .
\end{aligned}
$$

By an integration by parts, we have

$$
\begin{gathered}
\int_{\mathbb{R}} e^{i \varepsilon x \xi}\left(\sin \xi-\sin \left(\frac{\xi}{\sqrt{1+\varepsilon^{2} \xi^{2}}}\right)\right) \frac{\sqrt{1+\varepsilon^{2} \xi^{2}}}{\xi} \widehat{\psi}\left(\varepsilon^{1-\theta} \xi\right) d \xi \\
=\int_{0}^{1} \int_{\mathbb{R}} \cos \left(\lambda \xi+(1-\lambda) \frac{\xi}{\sqrt{1+\varepsilon^{2} \xi^{2}}}\right) \\
\quad \times\left(\xi-\frac{\xi}{\sqrt{1+\varepsilon^{2} \xi^{2}}}\right) \frac{\sqrt{1+\varepsilon^{2} \xi^{2}}}{\xi} e^{i \varepsilon x \xi} \widehat{\psi}\left(\varepsilon^{1-\theta} \xi\right) d \xi d \lambda \\
=-\int_{0}^{1} \int_{\mathbb{R}} \sin \left(\lambda \xi+(1-\lambda) \frac{\xi}{\sqrt{1+\varepsilon^{2} \xi^{2}}}\right) \\
\quad \times \frac{\partial}{\partial \xi}\left(\frac{\left(\xi-\frac{\xi}{\sqrt{1+\varepsilon^{2} \xi^{2}}}\right) \frac{\sqrt{1+\varepsilon^{2} \xi^{2}}}{\lambda} e^{i \varepsilon x \xi} \widehat{\psi}\left(\varepsilon^{1-\theta} \xi\right)}{\lambda+\frac{(1-\lambda)}{\left(1+\varepsilon^{2} \xi^{2}\right)^{\frac{3}{2}}}}\right) d \xi d \lambda \\
=o(1) \quad \text { as } \quad \varepsilon \rightarrow 0 \quad \text { uniformly on compact subsets of } \mathbb{R} .
\end{gathered}
$$

We also have

$$
\int_{\mathbb{R}} e^{i \varepsilon x \xi} \frac{\sin \xi}{\xi}\left(\sqrt{1+\varepsilon^{2} \xi^{2}}-1\right) \widehat{\psi}\left(\varepsilon^{1-\theta} \xi\right) d \xi=o(1) \quad \text { as } \quad \varepsilon \rightarrow 0
$$

uniformly on compact subsets of $\mathbb{R}$. From these two estimates, we deduce that it suffices to show

$$
\lim _{\varepsilon \rightarrow 0} \frac{1}{2 \pi} \int_{\mathbb{R}} e^{i \varepsilon x \xi} \frac{\sin \xi}{\xi} \widehat{\psi}\left(\varepsilon^{1-\theta} \xi\right) d \xi=\pi .
$$

Since $\frac{\sin \xi}{\xi}=\frac{1}{2 i} \int_{-1}^{1} e^{i \xi y} d y$ and $\int \psi d x=1$, we have

$$
\begin{aligned}
\frac{1}{2 \pi} \int_{\mathbb{R}} e^{i \varepsilon x \xi} \frac{\sin \xi}{\xi} \widehat{\psi}\left(\varepsilon^{1-\theta} \xi\right) d \xi & =\frac{1}{4 \pi i} \int_{-1}^{1} \int_{\mathbb{R}} e^{i \xi(\varepsilon x+y)} \widehat{\psi}\left(\varepsilon^{1-\theta} \xi\right) d \xi d y \\
& =\varepsilon^{-(1-\theta)} \frac{1}{2 i} \int_{-1}^{1} \psi\left(\frac{\varepsilon x+y}{\varepsilon^{1-\theta}}\right) d y \\
& =\frac{1}{2 i} \int_{-\frac{1}{\varepsilon^{1-\theta}}-\varepsilon^{\theta} x}^{\frac{1}{\varepsilon^{1-\theta}}-\varepsilon^{\theta} x} \psi(y) d y \rightarrow \frac{1}{2 i} \text { as } \quad \varepsilon \rightarrow 0
\end{aligned}
$$

uniformly on compact subsets of $\mathbb{R}$. This completes the proof of (2.5). 


\section{Proof of the Theorems}

3.1. Existence and scattering. The strategy of proof is to use the standard contraction mapping theorem. For this purpose, let us define a nonlinear mapping $\mathcal{N}$ by

$$
\mathcal{N}(u)=\partial_{t} S(t) \varphi+S(t) \psi+\int_{0}^{t} T\left(t-t^{\prime}\right) f(u)\left(t^{\prime}\right) d t^{\prime}
$$

We will prove that for sufficiently small $\rho, \mathcal{N}$ maps from $X_{\rho}^{s, \theta}$ to $X_{\rho}^{s, \theta}$. To do this, we introduce generalized chain and Leibniz rules:

Lemma 3.1. For any $s \geq 0$, we have

$$
\begin{aligned}
& \left\|D^{s} f(u)\right\|_{L^{r}} \lesssim\|u\|_{L^{(p-1) r_{1}}}^{p-1}\left\|D^{s}\right\|_{L^{r_{2}}}, \\
& \left(\frac{1}{r}=\frac{1}{r_{1}}+\frac{1}{r_{2}}, \quad r_{1} \in(1, \infty], r_{2} \in(1, \infty)\right) \\
& \left\|D^{s}(u v)\right\|_{L^{r}} \lesssim\left\|D^{s} u\right\|_{L^{r_{1}}}\|v\|_{L^{q_{2}}}+\|u\|_{L^{q_{1}}}\left\|D^{s} v\right\|_{L^{r_{2}}} . \\
& \left(\frac{1}{r}=\frac{1}{r_{1}}+\frac{1}{q_{2}}=\frac{1}{q_{1}}+\frac{1}{r_{2}}, \quad r_{i} \in(1, \infty), q_{i} \in(1, \infty] \quad(i=1,2)\right)
\end{aligned}
$$

We should emphasize that the exponents $r_{1}$ of $(3.1), q_{1}, q_{2}$ of (3.2) can be infinite. One can easily prove the lemma above by following and modifying slightly the proof of Proposition 3.1 and 3.3 in [9]. Also see the appendix of [14].

Now let $s>2-\frac{4}{r}$ and $\theta=\min \left(\frac{1}{2}-\frac{1}{r}, \frac{4}{r}\right)$. Then from Lemma $2.2-2.4$, the condition (1.3) and the chain rule (3.1), it follows that for any $u \in X_{\rho}^{s, \theta}$

$$
\begin{aligned}
\|\mathcal{N}(u)\|_{L^{\infty}} & \\
& \lesssim(1+t)^{-\theta} \delta+\int_{0}^{t}\left(1+t-t^{\prime}\right)^{-\theta}\left(\|f(u)\|_{L^{1}}+\left\|D^{s} f(u)\right\|_{L^{r^{\prime}}}\right) d t^{\prime} \\
& \lesssim(1+t)^{-\theta} \delta+\int_{0}^{t}\left(1+t-t^{\prime}\right)^{-\theta}\left(\|u\|_{L^{\infty}}^{p-2}\|u\|_{L^{2}}^{2}+\|u\|_{L^{\frac{2(p-1) r}{r-2}}}^{p-1}\left\|D^{s} u\right\|_{L^{2}}\right) d t^{\prime} \\
& \lesssim(1+t)^{-\theta} \delta+\int_{0}^{t}\left(1+t-t^{\prime}\right)^{-\theta}\left(\|u\|_{L^{\infty}}^{p-2}\|u\|_{L^{2}}^{2}+\|u\|_{L^{\infty}}^{p-2+\frac{2}{r}}\|u\|_{L^{2}}^{1-\frac{2}{r}}\left\|D^{s} u\right\|_{L^{2}}\right) d t^{\prime} \\
& \lesssim(1+t)^{-\theta} \delta+\rho^{p} \int_{0}^{t}\left(1+t-t^{\prime}\right)^{-\theta}\left(1+t^{\prime}\right)^{-(p-2) \theta} d t^{\prime}
\end{aligned}
$$

Since $(p-2) \theta>1$, we have for sufficiently small $\delta$ and $\rho$

$$
\sup _{t>0}(1+t)^{\theta}\|\mathcal{N}(u)\|_{L^{\infty}} \lesssim \delta+\rho^{p}<\frac{\rho}{2}
$$


And also we have

$$
\begin{aligned}
\|\mathcal{N}(u)\|_{H^{s}} \lesssim\|\varphi\|_{H^{s}}+\left\|D^{-1} \psi\right\|_{L^{2}}+\|\psi\|_{H^{s}}+\int_{0}^{t}\|f(u)\|_{H^{s}} d t^{\prime} \\
\lesssim\|\varphi\|_{H^{s}}+\left\|D^{-1} \psi\right\|_{L^{2}}+\|\psi\|_{H^{s}} \\
\left.\quad+\int_{0}^{t}\left(\|u\|_{L^{2 p}}^{p}+\|u\|_{L^{\infty}}^{p-1} \| D^{s} u\right) \|_{L^{2}}\right) d t^{\prime} \\
\\
\quad \lesssim \delta+\rho^{p}<\frac{\rho}{2}
\end{aligned}
$$

Thus $\mathcal{N}$ maps from $X_{\rho}^{s, \theta}$ to $X_{\rho}^{s, \theta}$.

Now for any $u, v \in X_{\rho}^{s, \theta}$ we can show from the chain rule (3.1) and Leibniz rule (3.2) that if $\delta$ and $\rho$ are sufficiently small, then

$$
\begin{aligned}
& \|\mathcal{N}(u)-\mathcal{N}(v)\|_{L^{\infty}} \\
& \lesssim \int_{0}^{t}\left(1+t-t^{\prime}\right)^{-\theta}\left(\|f(u)-f(v)\|_{L^{1}}+\left\|D^{s}(f(u)-f(v))\right\|_{L^{r^{\prime}}} d t^{\prime}\right. \\
& \lesssim \int_{0}^{t}\left(1+t-t^{\prime}\right)^{-\theta}\left(\left(\|u\|_{L^{\infty}}^{p-3}\|u\|_{L^{2}}^{2}+\|v\|_{L^{\infty}}^{p-3}\|v\|_{L^{2}}^{2}\right)\|u-v\|_{L^{\infty}}\right. \\
& +\left(\|u\|_{L}^{p-1} \frac{2(p-1) r}{r-2}+\|v\|_{L}^{p-1} \frac{2(p-1) r}{r-2}\right)\left\|D^{s}(u-v)\right\|_{L^{2}} \\
& \left.+\left(\|u\|_{L^{\frac{2(p-2) r}{r-2}}}^{p-2}+\|v\|_{L^{\frac{2(p-2) r}{r-2}}}^{p-2}\right)\left(\left\|D^{s} u\right\|_{L^{2}}+\left\|D^{s} v\right\|_{L^{2}}\right)\|u-v\|_{L^{\infty}}\right) d t^{\prime} \\
& \lesssim \rho^{p-1}|| \mid u-v\|\|_{s, \theta} \int_{0}^{t}\left(1+t-t^{\prime}\right)^{-\theta}\left(1+t^{\prime}\right)^{-(p-2) \theta} d t^{\prime} \\
& \lesssim(1+t)^{-\theta} \rho^{p-1}\left\||u-v \||_{s, \theta}\right.
\end{aligned}
$$

Similarly, we can also show

$$
\|\mathcal{N}(u)-\mathcal{N}(v)\|_{H^{s}} \lesssim \rho^{p-1}\|\| u-v \|_{s, \theta}
$$

Thus for small $\rho, \mathcal{N}$ is a contraction mapping and hence there exists a unique solution $u \in X_{\rho}^{s, \theta}$ to the problem $\mathcal{N}(u)=u$.

Since the time derivative $u_{t}$ satisfies the following equation:

$$
\begin{aligned}
u_{t}(x, t)= & -\frac{1}{2 \pi} \int_{\mathbb{R}} e^{i x \xi} \sin \left(\frac{t \xi}{\sqrt{1+\xi^{2}}}\right) \frac{\xi}{\sqrt{1+\xi^{2}}} \widehat{\varphi} d \xi \\
& +\frac{1}{2 \pi} \int_{\mathbb{R}} e^{i x \xi} \cos \left(\frac{t \xi}{\sqrt{1+\xi^{2}}}\right) \widehat{\psi} d \xi \\
& +\frac{1}{2 \pi} \int_{0}^{t} \int_{\mathbb{R}} e^{i x \xi} \cos \left(\frac{\left(t-t^{\prime}\right) \xi}{\sqrt{1+\xi^{2}}}\right) \frac{\xi^{2}}{1+\xi^{2}} \widehat{f(u)}\left(\xi, t^{\prime}\right) d \xi d t^{\prime},
\end{aligned}
$$

by the same argument in Section 2 , one can easily show that $u_{t} \in X_{\rho}^{s, \theta}$, provided $\delta$ and $\rho$ are much smaller. This completes the proof of Theorem 1.1. 
Once the existence has been established, the proof of Theorem 1.2 is rather straight forward. Let us define functions $\varphi^{+}$and $\psi^{+}$by

$$
\begin{aligned}
& \widehat{\varphi^{+}}(\xi)=\widehat{\varphi}(\xi)+\int_{0}^{\infty} \frac{\xi}{\sqrt{1+\xi^{2}}} \sin \frac{t \xi}{\sqrt{1+\xi^{2}}} \widehat{f(u)}(\xi, t) d t \\
& \widehat{\psi^{+}}(\xi)=\widehat{\psi}(\xi)-\int_{0}^{\infty} \frac{\xi^{2}}{1+\xi^{2}} \cos \frac{t \xi}{\sqrt{1+\xi^{2}}} \widehat{f(u)}(\xi, t) d t .
\end{aligned}
$$

Let $u^{+}$be the solution to the linear problem (1.4) with initial data $\left(\varphi^{+}, \psi^{+}\right)$. Then it can be represented by

$$
u^{+}(x, t)=\left(\partial_{t} S(t) \varphi\right)(x)+(S(t) \psi)(x)+\int_{0}^{\infty} T\left(t-t^{\prime}\right) f\left(u\left(t^{\prime}\right)\right) d t^{\prime} .
$$

Since $u, u_{t} \in X_{\rho}^{s, \theta}$ for $s>2-\frac{4}{r}$ and $\theta=\min \left(\frac{r-2}{2 r}, \frac{4}{r}\right)$, we have from Lemma 3.2

$$
\begin{aligned}
\left\|u(\cdot, t)-u^{+}(\cdot, t)\right\|_{H^{s}} & \lesssim \int_{t}^{\infty}\left\|f\left(u\left(\cdot, t^{\prime}\right)\right)\right\|_{H^{s}} d t^{\prime} \lesssim \rho^{p} \int_{t}^{\infty}\left(1+t^{\prime}\right)^{-\theta(p-1)} d t^{\prime} \\
& =O\left(t^{-\theta(p-1)+1}\right) .
\end{aligned}
$$

Similarly, we have

$$
\left\|u_{t}(\cdot, t)-u_{t}^{+}(\cdot, t)\right\|_{H^{s}} \lesssim \int_{t}^{\infty}\left\|f(u)\left(t^{\prime}\right)\right\|_{H^{s}} d t^{\prime}=O\left(t^{-\theta(p-1)+1}\right) .
$$

Since $\theta(p-1)>1$, we have just proved the theorem. For more details, see [7].

3.2. Nonexistence of nontrivial asymptotically free solutions. Let us define a bilinear form $H(u, v)(t)$ by

$$
H(u, v)(t)=\operatorname{Re} \int_{\mathbb{R}}\left(D^{-1} u_{t}(t) \overline{D^{-1} v(t)}-D^{-1} v_{t}(t) \overline{D^{-1} u}(t)\right) d x .
$$

Then $H(u, v)(t)$ is well-defined and uniformly bounded on $t>0$ for $\left(u, u_{t}\right),\left(v, v_{t}\right) \in$ $L^{\infty}\left(0, \infty ; D L^{2} \times D^{2} L^{2}\right)$.

Our strategy of proof is to use a contradiction to the uniform boundedness of $H$. Suppose that there are non-zero functions $u$ and $u^{+}$satisfying the condition of Theorem 1.3. Then we obtain

$$
\frac{d}{d t} H\left(u, u^{+}\right)(t)=\operatorname{Re} \int f(u) \overline{u^{+}} d x .
$$

Let $H\left(u, u^{+}\right)(t)=H(t)$. Then we have

$$
\begin{aligned}
\frac{d}{d t} H(t) & =\operatorname{Re} \int\left(f(u)-f\left(u^{+}\right)\right) \overline{u^{+}} d x+\operatorname{Re} \int f\left(u^{+}\right) \overline{u^{+}} d x \\
& \geq \operatorname{Re} \int\left(f(u)-f\left(u^{+}\right)\right) \overline{u^{+}} d x+c \int\left|u^{+}\right|^{p+1} d x
\end{aligned}
$$

Now using an argument in [1] and [12], we prove that if $t$ is sufficiently large,

$$
\left\|u^{+}(t)\right\|_{L^{p+1}\left(|x| \leq A t^{\beta}\right)}^{p+1} \geq c_{0} t^{-\beta \frac{p-1}{2}}
$$


for some positive constant $A$ and $c_{0}$ depending on $\varphi^{+}$and $D^{-1} \psi^{+}$and $\beta>1$ depending on $\varepsilon$. Here and after, every constant depends on $\varphi^{+}$and $\psi^{+}$, if not specified. For the proof of (3.6), we first show that

$$
\left\|u^{+}(t)\right\|_{L^{2}\left(|x| \leq A t^{\beta}\right)} \gtrsim 1 \quad \text { for sufficiently large } t .
$$

Using Hölder inequality, (3.7) yields the required estimate (3.6). To obtain the $L^{2}$ lower bound, let us choose a cut off function $\chi_{0}$ supported in $(-1,1)$ such that

$$
\left\|u^{+}(t)\right\|_{L^{2}\left(|x| \leq A t^{\beta}\right)}^{2}=t\left\|u^{+}(t \cdot, t)\right\|_{L^{2}(|x| \leq M)}^{2} \geq t\left\|\chi_{0}(\cdot / M) u^{+}(t \cdot, t)\right\|_{L^{2}}^{2},
$$

where $M=A t^{\beta-1}$. Since $u^{+}$is the solution to the linear problem (1.4), for the last integral, we have

$$
\begin{aligned}
& t\left\|\chi_{0}(\cdot / M) u^{+}(t \cdot, t)\right\|_{L^{2}}^{2} \\
& =t\left\|\chi_{0}(\cdot / M)\left(\partial_{t} S(t) \varphi^{+}\right)(t \cdot)\right\|_{L^{2}}^{2}+t\left\|\chi_{0}(\cdot / M)\left(S(t) \psi^{+}\right)(t \cdot)\right\|_{L^{2}}^{2} \\
& \quad+2 t \operatorname{Re} \int\left(\chi_{0}(x / M)\right)^{2}\left(\partial_{t} S(t) \varphi^{+}\right)(t x) \overline{\left(S(t) \psi^{+}\right)(t x)} d x .
\end{aligned}
$$

By change of variable and Plancheral's theorem, we have for the first term

$$
t\left\|\chi_{0}(\cdot / M)\left(\partial_{t} S(t) \varphi^{+}\right)(t \cdot)\right\|_{L^{2}}^{2}=\left\|\chi_{0}(\cdot / M) \mathcal{F}^{-1}\left(\cos \frac{(\cdot)}{\sqrt{1+(\cdot)^{2} / t^{2}}} t^{-\frac{1}{2}} \widehat{\varphi^{+}}(\cdot / t)\right)\right\|_{L^{2}}^{2} .
$$

From the identity $\cos ^{2} x=\frac{1+\cos (2 x)}{2}$, we deduce that

$$
\begin{aligned}
& \left\|\cos \frac{(\cdot)}{\sqrt{1+(\cdot)^{2} / t^{2}}} t^{-\frac{1}{2}} \widehat{\varphi^{+}}(\cdot / t)\right\|_{L^{2}}^{2} \\
& \quad=\int \cos ^{2}\left(\frac{\xi}{\sqrt{1+\xi^{2} / t^{2}}}\right) t^{-1} \widehat{\varphi^{+}}(\xi / t) \widehat{\widehat{\varphi^{+}}}(\xi / t) d \xi \\
& \quad=\frac{1}{2}\left\|\varphi^{+}\right\|_{L^{2}}^{2}+\frac{1}{2} \int \cos \left(\frac{2 \xi}{\sqrt{1+\xi^{2} / t^{2}}}\right) t^{-1} \widehat{\varphi^{+}}(\xi / t) \widehat{\widehat{\varphi^{+}}}(\xi / t) d \xi .
\end{aligned}
$$

By the integration by parts, it follows from the Hölder inequality that

$$
\begin{aligned}
& \int \cos \left(\frac{2 \xi}{\sqrt{1+\xi^{2} / t^{2}}}\right) t^{-1} \widehat{\varphi^{+}}(\xi / t) \widehat{\widehat{\varphi^{+}}}(\xi / t) d \xi \\
& =-\frac{1}{2 t} \int \sin \left(\frac{2 \xi}{\sqrt{1+\xi^{2} / t^{2}}}\right) \partial_{\xi}\left(\left(1+\xi^{2} / t^{2}\right)^{\frac{3}{2}} \widehat{\varphi^{+}}(\xi / t) \overline{\widehat{\varphi^{+}}}(\xi / t)\right) d \xi \\
& =O\left(t^{-1}\right)
\end{aligned}
$$

and hence

$$
\left\|\left(\cos \frac{(\cdot)}{\sqrt{1+(\cdot)^{2} / t^{2}}}\right) t^{-\frac{1}{2}} \widehat{\varphi^{+}}(\cdot / t)\right\|_{L^{2}} \rightarrow \frac{1}{\sqrt{2}}\left\|\varphi^{+}\right\|_{L^{2}} \quad \text { as } \quad t \rightarrow \infty .
$$

Now we claim that there exist large numbers $t_{0}$ such that

$$
\inf _{t>t_{0}} t\left\|\chi_{0}(\cdot / M)\left(\partial_{t} S(t) \varphi^{+}\right)(t \cdot)\right\|_{L^{2}}^{2} \gtrsim 1 .
$$


For the proof of (3.11), we may assume that $\left\|\varphi^{+}\right\|_{L^{2}}=1$. Let us define a function $g_{t}(x)$ by $t\left|\left(\partial_{t} S(t) \varphi^{+}\right)(t x)\right|^{2}$. Then from (3.10), we can find a positive number $t_{0}$ such that $\left\|g_{t}\right\|_{L^{1}} \geq \frac{1}{2}$ for all $t>t_{0}$. Using the integration by parts $m$-times, we get for $x \neq 0$

$$
\begin{aligned}
t^{\frac{1}{2}}\left(\partial_{t} S(t) \varphi^{+}\right)(t x) & =\frac{1}{2 \pi} \int e^{i x \xi} \cos \left(\frac{\xi}{\sqrt{1+\xi^{2} / t^{2}}}\right) t^{-\frac{1}{2}} \widehat{\varphi^{+}}(\xi / t) d \xi \\
& =\frac{1}{2 \pi(-i x)^{m}} \int e^{i x \xi} \partial_{\xi}^{m}\left(\cos \left(\frac{\xi}{\sqrt{1+\xi^{2} / t^{2}}}\right) t^{-\frac{1}{2}} \widehat{\varphi^{+}}(\xi / t)\right) d \xi
\end{aligned}
$$

We then have $g_{t}(x) \leq \frac{A t}{|x|^{2 m}}$ for some $A$ depending on $\varphi^{+}$. This gives us that

$$
\begin{aligned}
\int\left(\chi_{0}(x / M)\right)^{2} g_{t}(x) d x & =\int g_{t}(x) d x-\int\left(1-\left(\chi_{0}^{2}(x / M)\right)^{2}\right) g_{t}(x) d x \\
& \geq \frac{1}{2}-\int_{|x| \geq \frac{1}{2} M} \frac{A t}{|x|^{2 m}} d x \\
& \geq \frac{1}{2}-\frac{2}{2 m-1} A t\left(1 / 2 A t^{\beta-1}\right)^{1-2 m}
\end{aligned}
$$

Now if we choose $m$ and $\beta$ so that $(\beta-1)(2 m-1)>1$, then the claim (3.11) is proved, provided $t_{0}$ is sufficiently large.

Similarly we can prove that

$$
\left\|\left(\sin \frac{(\cdot)}{\sqrt{1+(\cdot)^{2} / t^{2}}}\right) \frac{\sqrt{1+\xi^{2} / t^{2}}}{\xi / t} t^{-\frac{1}{2}} \widehat{\psi^{+}}(\cdot / t)\right\|_{L^{2}}^{2} \rightarrow \frac{1}{\sqrt{2}}\left\|\left(1-\partial_{x}^{2}\right)^{\frac{1}{2}} D^{-1} \psi^{+}\right\|_{L^{2}}^{2}
$$

as $t \rightarrow \infty$ and hence by the same argument as above, we have the estimate

$$
t\left\|\chi_{0}(\cdot / M)\left(S(t) \psi^{+}\right)(t \cdot)\right\|_{L^{2}}^{2} \gtrsim 1
$$

if $t>t_{0}$ for some large $t_{0}$.

Finally, for the last term of (3.8) let us consider the integral

$$
I(t)=t \int\left(\partial_{t} S(t) \varphi^{+}\right)(t x) \overline{S(t) \psi^{+}}(t x) d x .
$$

Then by change of variable and Plancheral's theorem, $I(t)$ is converted by

$$
\frac{1}{2 t \pi} \int \sin \left(\frac{2 \xi}{\sqrt{1+\xi^{2} / t^{2}}}\right) \frac{\sqrt{1+\xi^{2} / t^{2}}}{2 \xi / t} \widehat{\varphi^{+}}(\xi / t) \widehat{\psi}(\xi / t) d \xi
$$

Here we also used the identity $\cos x \sin x=\frac{1}{2} \sin 2 x$. Similarly to the estimate (3.9), we have $I(t)=O\left(t^{-1}\right)$. With this estimate we prove that

$$
\left|2 t \operatorname{Re} \int\left(\chi_{0}(x / M)\right)^{2}\left(\partial_{t} S(t) \varphi^{+}\right)(t x) \overline{\left(S(t) \psi^{+}\right)(t x)} d x\right| \rightarrow 0 \quad \text { as } \quad t \rightarrow \infty .
$$


Actually, by the integration by parts as above, we have

$$
\begin{aligned}
& \left|2 t \operatorname{Re} \int\left(\chi_{0}(x / M)\right)^{2}\left(\partial_{t} S(t) \varphi^{+}\right)(t x) \overline{\left(S(t) \psi^{+}\right)(t x)} d x\right| \\
& \quad \leq|2 \operatorname{Re} I(t)|+\int_{|x| \geq \frac{1}{2} M} \frac{A t}{|x|^{2 m}} d x \rightarrow 0
\end{aligned}
$$

as $t \rightarrow \infty$.

Therefore (3.13) together with (3.11) and (3.12) yields the lower bound estimate (3.7) and hence (3.6).

Since $\varphi^{+}$and $\psi^{+}$are in $\mathcal{F}^{-1} C_{0}^{\infty}(\mathbb{R} \backslash\{0\})$, it follows from the proof of Lemmas 2.2 and 2.3 that for all $2 \leq q \leq \infty$

$$
\left\|u^{+}(t)\right\|_{L^{q}} \lesssim t^{-\left(\frac{1}{2}-\frac{1}{q}\right)}
$$

From the estimate (3.14) and the hypothesis (1.5), we readily have for $1<p \leq 2$

$$
\begin{aligned}
& \left|\operatorname{Re} \int\left(f(u)-f\left(u^{+}\right)\right) \overline{u^{+}} d x\right| \\
& \quad \lesssim\left(\|u\|_{L^{2}}^{p-1}\left\|u^{+}\right\|_{L^{2}}^{2-p}+\left\|u^{+}\right\|_{L^{2}}\right)\left\|u^{+}\right\|_{L^{\infty}}^{p-1}\left\|u-u^{+}\right\|_{L^{2}} \\
& \quad=O\left(t^{-\frac{1}{2}(p-1)-\varepsilon}\right) .
\end{aligned}
$$

Thus choosing $\beta$ such as $\frac{\beta(p-1)}{2}<\frac{p-1}{2}+\varepsilon$ and $\frac{\beta(p-1)}{2} \leq 1$, we conclude from (3.6) that $\frac{d}{d t} H(t) \gtrsim t^{-1}$ for large $t$. This is a contradiction to the uniform boundedness of $H$.

3.3. Remarks. The methods of proof for Theorems 1.1 and 1.2 are applicable to the high dimensional case with a slight modification of Lemma 2.1. One can treat the high dimensional version of Lemma 2.1 by using a dyadic decomposition and the method of stationary phase in the case of non-vanishing Gaussian curvature of the phase. In our problem, since the phase $\omega=\frac{|\xi|}{\sqrt{1+|\xi|^{2}}}$ is radially symmetric, the Gaussian curvature of $\omega$ is equivalent to the value of second derivative of $\frac{r}{\sqrt{1+r^{2}}}(r=$ $|\xi|)$. Thus we can easily obtain the high dimensional analogs of Theorems 1.1 and 1.2. As for the Theorem 1.3, using the radial symmetry, one can carry out the integration by parts with respect to the radial derivatives and hence obtain a high dimensional version of (3.7). Then by a straightforward application of the one dimensional argument, one can have the nonexistence of scattering for $1<p \leq 1+\frac{2}{n}$ like Schrödinger or Klein-Gordon equation.

In the proof of Theorem 1.3, the assumption $\left\|u(t)-u^{+}(t)\right\|_{L^{2}}=O\left(t^{-\varepsilon}\right)$ was necessary for the comparison between (3.6) and (3.15). For the proof of (3.6), it was inevitable to use $M=A t^{\beta-1}$ unlike the Schrödinger case in [1] where $M$ is just a large constant. In our problem, the dependence of $M$ on $t$ was caused by the reason that the norm $\left\|t^{\frac{1}{2}} \chi_{0}(\cdot / M) \partial_{t} S(t) \varphi^{+}(t \cdot)\right\|_{L^{2}}^{2}$ converges to the norm $\frac{1}{2}\left\|\varphi^{+}\right\|_{L^{2}}^{2}$ but the function $t^{\frac{1}{2}} \chi_{0}(\cdot / M) \partial_{t} S(t) \varphi^{+}(t \cdot)$ itself does not converges to $\frac{1}{\sqrt{2}} \varphi^{+}$in $L^{2}$ 
because the phase $\omega=\frac{\xi}{\sqrt{1+\xi^{2}}}$ is almost constant at high frequency. This is a difficulty different from other dispersive equations with well curved phase $\omega$ like the Schrödinger case $\omega=|\xi|^{2}$ and so on. It will be very interesting to prove the nonexistence of scattering without decay assumption (1.5).

\section{REFERENCES}

[1] Barab, J. E. 1984 Nonexistence of asymptotically free solutions for a nonlinear Schrödinger equation, J. Math. Phys. 25, 3270-3274.

[2] Bergh, J. \& Löfström, J. 1976 Interpolation Sapces, Springer-Verlag, New York.

[3] Boussinesq, M. J. 1877 Essai sur la théorie des eaux courantes, memo presented at the Académic des Sciences Inst. France(series), 23 pp. 1-680.

[4] Chen, G. \& Wang, S. 1998 Cauchy problem for generalized IMBq equation, in "Proceedings of the Conference on Nonlinear Partial Differential Equations and Applications," World Scientific, Singapore.

[5] Chen, G. \& Wang, S. 1999 Existence and nonexistence of global solutions for generalized IMBq equation, Nonlinear Anal. 36, 961-980.

[6] Chen, G. \& Wang, S. 2002 The Cauchy problem for the generalized IMBq equation in $W^{s, p}\left(\mathbb{R}^{n}\right)$, J. Math. Anal. Appl. 266, 38-54.

[7] Chen, G. \& Wang, S. 2002 Small amplitude solutions of the generalized IMBq equation, $J$. Math. Anal. Appl. 274, 846-866.

[8] Chen, G., Xing, J. \& Yang, Z. 1996 Cauchy problem for generalized IMBq equation with several variables, Nonlinear Anal. 26, 1255-1270.

[9] Christ, F. M. \& Weinstein, M. I. 1991 Dispersion of small amplitude solution of the generalized Korteweg-de Vries equation, J. Func. Anal. 100, 87-109.

[10] Clarkson, A., LeVeque, R. J. \& Saxton, R. 1986 Solitary-wave interaction in elastic rods, Stud. Appl. Math. 75, 95-122.

[11] Glassey, R. T. 1973 On the symptotic behavior of nonlinear wave equation, Trans. Amer. Math. Soc. 182, 187-200.

[12] Glassey, R. T. 1977 Asymptotic behavior of solutions to certain nonlinear Schrödinger-Hartree equations, Commun. Math. Phys. 53, 9-18.

[13] Kano, T. \& Nishida, T. 1986 A mathematical justification for Korteweg-de Vries equation and Boussinesq equation of water surface waves, Osaka J. Math. 23, 389-413.

[14] Kato, T. 1995 On nonlinear Schrödinger equations II. $H^{s}$-solutions and unconditional wellposedness, J. Anal. Math. 67, 281-306.

[15] Liu, Y. 1996 Existence and blow up of solutions of a nonlinear Pochhammer-Chree equation, Indiana Univ. Math. J. 45, 797-816.

[16] Makhankov, V. G. 1978 Dynamics of classical solitons (in non-integrabla systems), physics reports, Phys. Lett. C 35, 1-128.

[17] Matsumura, A. 1976 On the Asymptotic behavior of solutions of semi-linear wave equations, Publ. RIMS, Kyoto Univ. 12, 169-189.

[18] Stein, E. M. 1993 Harmonic Aanlysis, Princeton University Press, New Jersey.

[19] Strauss, W. A. 1989 Nonlinear Wave Equations, CBMS, Regional Conference Series in Mathematics no. 73 AMS.

Department of Mathematics, Hokkaido University, Sapporo 060-0810, Japan

E-mail address: ygcho@math.sci.hokudai.ac.jp

E-mail address: ozawa@math.sci.hokudai.ac.jp 